PROCEEDINGS OF THE

AMERICAN MATHEMATICAL SOCIETY

Volume 33, Number 2, June 1972

\title{
CATEGORICAL SEMIGROUPS
}

\author{
F. R. MCMORRIS AND M. SATYANARAYANA
}

\begin{abstract}
ABSTRACr. The main purpose of this paper is to describe some properties of categorical semigroups, commutative semigroups which are categorical at zero, and determine the structure of commutative categorical semigroups. We also investigate whether Petrich's tree condition, for categorical semigroups which are completely semisimple inverse semigroups, is necessary for arbitrary categorical semigroups.
\end{abstract}

Introduction. An ideal $A$ of a semigroup $S$ is categorical if abceA $(a, b, c \in S)$ implies $a b \in A$ or $b c \in A$. If $S=S^{0}$ and 0 is a categorical ideal, then $S$ is categorical at zero. If every ideal of $S$ is categorical, then $S$ is called a categorical semigroup.

W. D. Munn [2] and M. Petrich [4] have found that the concept of categorical ideals plays an important role in the theory of partial homomorphisms, extensions of inverse semigroups, and 0-restricted homomorphic images of inverse semigroups. Petrich has shown that a completely semisimple inverse semigroup $S$ is categorical if and only if the set of idempotents $E(S)$ of $S$ is a tree. However, we show that there exist regular categorical semigroups in which $E(S)$ is not a tree.

In this paper, we initiate the characterization of arbitrary categorical semigroups by focusing our attention on the commutative case. In the first section we prove that if $S$ is a commutative categorical semigroup, then $E(S)$ is a tree.

In 2.2 , we obtain that a commutative semigroup which is categorical at zero is the 0-direct union of two categorical ideals, and in 2.5, 2.6, and 2.7 we determine a relationship between being categorical at zero and having zero divisors.

Finally in $\S 3$, we prove that a commutative semigroup is categorical if and only if it is an extension of a regular categorical semigroup by a null semigroup.

All terminology not given here can be found in [1]

Received by the editors July 12, 1971.

AMS 1970 subject classifications. Primary $20 \mathrm{M} 10$.

Key words and phrases. Categorical ideals, categorical semigroups, semigroups categorical at zero.

(c) American Mathematical Society 1972 
The authors would like to express their appreciation to Professor A. H. Clifford for reading an earlier version of this paper and suggesting improvements. We also thank the referee for his suggestions.

\section{Categorical semigroups.}

THEOREM 1.1. Let $S$ be a semigroup with identity. Then the following are equivalent.

(i) $S$ is categorical;

(ii) $S$ is a union of simple semigroups, and the ideals of $S$ form a chain under set inclusion.

Proof. It is easy to see that if $S$ has an identity, then an ideal $A$ of $S$ is categorical if and only if $A$ is prime. Hence the result follows from Theorems 5.1 and 5.2 of [3].

COROLlary 1.2. A commutative semigroup $S$ with identity is categorical if and only if $S$ is a union of groups in which the ideals form a chain under set inclusion.

EXAMPLE 1. A semigroup without identity may contain a categorical ideal that is not prime. Let $S=\langle a\rangle$ be the infinite cyclic semigroup generated by $a$. Then the ideal generated by $a^{2}$ is categorical but not prime.

The set of idempotents $E(S)$ of the semigroup $S$ is said to form a tree if for any $e, f, g \in E(S), e \geqq f$ and $e \geqq g$ implies that either $f \leqq g$ or $g \leqq f$, where $\leqq$ is the natural partial order on $E(S)$. One of the striking results for categorical semigroups is a result of Petrich (Theorem 2 of [4]) which states that a completely semisimple inverse semigroup is categorical if and only if its idempotents form a tree.

EXAMPLE 2. The right zero semigroup of order two with identity adjoined is an example of a regular categorical semigroup in which $E(S)$ is not a tree.

We shall now prove that this tree condition is necessary for some other classes of semigroups.

First we recall some notation. If $S$ is a semigroup and $a \in S$, we let $J(a)$ be the principal ideal generated by $a$, and $J_{a}=\{b \in S: J(a)=J(b)\}$. There is a partial order on the $\mathscr{J}$-classes given by $J_{a} \leqq J_{b}$ if and only if $J(a) \subseteq J(b)$.

Proposition 1.3. Let $S$ be a categorical semigroup in which the idempotents commute. If for any $e, f \in E(S), e<f$ implies that $J_{e}<J_{f}$, then $E(S)$ is a tree.

Proof. Assume $E(S)$ is not a tree. Then there exists $e, f, g \in E(S)$ such that $e>f$ and $e>g$, while $f$ and $g$ are incomparable. Since the idempotents commute, $f g$ is an idempotent with $f>f g$ and $g>f g$. Now $f e g \in J(f g)$ but $f e \notin J(f g)$ and $e g \notin J(f g)$ by hypothesis. This contradicts the fact that $S$ is categorical. Hence $E(S)$ is a tree. 
COROLlaRY 1.4 (Necessity of Theorem 2 in [4]). Let $S$ be a categorical semigroup that is a completely semisimple inverse semigroup. Then $E(S)$ is a tree.

Proof. It can easily be shown that if $S$ is a completely semisimple sem:group, then $e, f \in E(S)$ and $e<f$ imply that $J_{e}<J_{f}$.

COROLlARY 1.5. Let $S$ be a categorical semigroup. If the idempotents of $S$ commute and every right ideal of $S$ is two-sided, then $E(S)$ is a tree.

Proof. Let $e, f \in E(S)$ with $e<f$. Then clearly we have $J_{e} \leqq J_{f}$. Assume $J_{e}=J_{i}$. Then $f \in J(f)=J(e) \subseteq e S$, the inclusion holding since $e S$ by assumption is a two-sided ideal. Therefore ef $=f$, which implies the contradiction $f \leqq e$. Hence $J_{e}<J_{f}$ and we may apply the theorem.

COROLLARY 1.6. If $S$ is a commutative categorical semigroup, then $E(S)$ is a tree.

We recall that a commutative semigroup is regular (inverse) if and only if it is a semilattice of groups, and this is true if and only if it is a completely semisimple inverse semigroup. Hence using Petrich's result, the converse of 1.6 is true if we add on the condition that $S$ is regular. In fact, we note that a commutative regular semigroup is categorical if and only if it is a tree of abelian groups.

EXAMPLE 3. If $S$ is a commutative semigroup and $E(S)$ is a tree, then $S$ need not be categorical. Let $S=\left\{a, a^{2}, a^{3}, a^{4}\right\}$ be the cyclic semigroup of index 3 and period 2 . Then $E(S)=\left\{a^{4}\right\}$ is a tree but $\left\{a^{3}, a^{4}\right\}$ is an ideal that is not categorical.

If $S$ is a regular semigroup, not necessarily commutative, and $E(S)$ is a chain, then we prove in 1.7 that $S$ is a categorical semigroup. It is open as to whether this result is true if "chain" is replaced by "tree".

Proposition 1.7. Let $S$ be a regular semigroup in which $E(S)$ is a chain. Then $S$ is a categorical semigroup. In fact, every ideal of $S$ is prime.

Proof. Since a prime ideal is categorical it suffices to show that every ideal is prime. Let $I$ be an ideal and $a b \in I$ with $a, b \in S$. Since $S$ is regular, there exist $x, y \in S$ such that $a=a x a$ and $b=b y b$ with $x a, b y \in E(S)$. Thus $x a \leqq b y$ or $b y \leqq x a$. Assume $x a \leqq b y$. Then $(x a)(b y)=x a$ and we have

$$
a b y=(a x a)(b y)=a(x a)(b y)=a x a=a .
$$

This implies that $a \in I$ since $a b \in I$. Similarly $b y \leqq x a$ implies that $b \in I$. Hence $I$ is prime.

2. Commutative semigroups which are categorical at zero. Throughout this section we consider semigroups with zero. 
LEMMA 2.1. If $S$ is a commutative semigroup which is categorical at zero, then the following are true.

(i) Every nonzero nilpotent element of $S$ has index two.

(ii) If $x \in S$ is nilpotent, then $x s=0$ for all $s \in S$.

(iii) If $x \in x S$ for every $x \in S$, then $S$ contains no nonzero nilpotent elements.

Proof. Let $x \in S$ be a nonzero nilpotent and $n$ the least positive integer such that $x^{n}=0$. Assume $n>2$. Then $x x^{n-2} x=0$ implies $x^{n-1}=0$, a contradiction. To prove (ii), let $x \in S$ be nilpotent and $s \in S$. Then $0=x s=x s x$, which implies $x s=0$. Clearly (iii) follows from (ii).

THEOREM 2.2. Let $S$ be a commutative semigroup which is categorical at zero. Then $S$ is the 0-direct union of two categorical ideals $N$ and $L \cup 0$, where $N$ is the set of nilpotent elements and $L=\{x: x S \neq 0\}$. Furthermore, $N S=0$ and $L \cup 0$ is a subsemigroup of $S$ which is itself categorical at zero.

Proof. From 2.1, we need only show that $L \cup 0$ is a categorical ideal and that $N$ is categorical. To prove that $L \cup 0$ is an ideal, it suffices to show that $x, y \in S$ and $x y \in N$ imply $x y=0$. If $x y \in N$, then $x y x=0$ by (ii) of 2.1 . Hence $x y=0$ since 0 is categorical.

Now let $a b c \in L \cup 0$ where $a, b, c \in S$. If $a b c=0$, then $a b=0 \in L \cup 0$ or $b c=0 \in L \cup 0$. If $a b c \neq 0$, then we assert that $a, b, c \in L$. For instance if $a \notin L$, then $a \in N$ and by (ii) of 2.1 we would have $a(b c)=0$. Therefore $a b \in L \cup 0$ and we have that $L \cup 0$ is categorical.

To show that $N$ is categorical, let $a b c \in N$. As before, this implies that $a b c=0$. Hence $a b=0 \in N$ or $b c=0 \in N$.

COROLlaRY 2.3. Let $S$ be a commutative semigroup which is categorical at zero and such that $S=S^{2}$. Then either $S$ is a null semigroup or $S$ tas no nonzero nilpotent elements.

Proof. Assume $S$ not a null semigroup. Then $S=N \cup L$ with $L$ nonempty. Since $S=S^{2}, N^{2}=0$, and $N L=0$, we have $N \cup L=L^{2}$. Hence $N \subseteq L^{2} \subseteq L \cup 0$ and thus $N=0$.

We note that since the 0 -direct union of semigroups categorical at zero is also categorical at zero, Theorem 2.2 reduces the study of commutative semigroups categorical at zero to those having no nilpotent elements.

EXAMPLE 4. The converse to 2.2 need not be true. Let $S$ be the semilattice $\{1, e, f, 0\}$ where 1 is the identity, 0 is the zero, and $e f=f e=0$. Then $S=N \cup L$ as in 2.2 , but $S$ is not categorical at zero since $e l f=0$ with $e \neq 0$ and $f \neq 0$.

The 0 of a semigroup is said to be indecomposable if and only if the intersection of two nonzero ideals is nonzero. 
Proposition 2.4. Let $S$ be a commutative semigroup which is categorical at zero. If 0 is indecomposable, then

(i) There are no nonzero nilpotent elements, and for every nonzero $x \in S$, there exists $y \in S$ such that $x y \neq 0$, or

(ii) $S$ is the null semigroup of order two.

Proof. Let $S=N \cup L$ as given by 2.2 . Now $N \cap(L \cup 0)=0$ implies that $N=0$ or $L \cup 0=0$. If $N=0$, then $S=L \cup 0$ and we have (i).

If $L \cup 0=0$, then $N=S$. Assume $S$ contains two distinct nonzero elements $a$ and $b$. This implies $\{0, a\}$ and $\{0, b\}$ are nonzero ideals intersecting at 0 , which is a contradiction. Thus (ii) follows in this case.

If a semigroup has no zero-divisors, then evidently $S$ is categorical at zero. However the semilattice $\{e, f, 0\}$ with $e f=0$ is categorical at zero having zero-divisors.

The following is evident.

Proposition 2.5. Let $S$ be a commutative semigroup with identity. Then $S$ has no zero-divisors if and only if $S$ is categorical at zero.

THEOREM 2.6. Let $S$ be a commutative semigroup in which the ideals form a chain under set inclusion. Then $S$ is categorical at zero if and only if $S$ has no zero-divisors or $S$ is the null semigroup of order two.

Proof. Assume $S$ is categorical at zero and $S=N \cup L$ as in 2.2. Since the ideals form a chain, we have $N \subseteq L \cup 0$ or $L \cup 0 \subseteq N$. Thus $S=N$ or $S=L \cup 0$. If $S=N$, then $S$ is the null semigroup of order two as in 2.4, since the fact that the ideals form a chain implies that 0 is indecomposable.

Let $S=L \cup 0$. Assume $x y=0$ for some $x, y \in L$. Let $x^{R}$ and $y^{R}$ denote the annihilators of $x$ and $y$. Hence $x^{R} \subseteq y^{R}$ or $y^{R} \subseteq x^{R}$. In the first case, $y \in x^{R}$ implies $y \in y^{R}$ and thus $y^{2}=0$. This contradicts the fact that $L$ contains no nonzero nilpotent elements. Similarly the second case implies a contradiction. Therefore $S$ has no zero-divisors.

The converse is obvious.

By noting that the ideals form a chain in a commutative semigroup in which every ideal is principal [5], we have the following.

COROLlary 2.7. Let $S$ be a commutative semigroup in which every ideal is principal. Then $S$ is categorical at zero if and only if $S$ has no zerodivisors, or $S$ is the null semigroup of order two.

\section{Commutative categorical semigroups.}

Proposition 3.1. Let $S$ be a commutative categorical semigroup. Then the following are true.

(i) If $a \in S$, then $a^{2}=a^{3}$ or $a^{2}=a^{3} x$ for some $x \in S$.

(ii) If $I$ is an ideal of $S$, then $I^{2}=I^{3}$.

(iii) $S$ contains idempotents. 
Proof. (i) If $a \in S$, then $a^{3} \in a^{3} \cup a^{3} S$. Since $a^{3} \cup a^{3} S$ is categorical, we have $a^{2} \in a^{3} \cup a^{3} S$.

(ii) Let $a, b \in I$. Then $a b a \in I^{3}$, which implies that $a b \in I^{3}$ since $I^{3}$ is categorical. Thus $I^{2} \subseteq I^{3}$ and hence $I^{2}=I^{3}$.

(iii) Let $a \in S$. Then (i) implies $a^{2}=a^{3}$ or $a^{2}=a^{3} x$ for some $x \in S$. In the first case, $a^{2} \in E(S)$. If $a^{2}=a^{3} x$, then

$$
\left(a^{2} x^{2}\right)\left(a^{2} x^{2}\right)=\left(a^{3} x\right)\left(a x^{3}\right)=a^{2}\left(a x^{3}\right)=a^{3} x^{3}=\left(a^{3} x\right) x^{2}=a^{2} x^{2} .
$$

Hence $a^{2} x^{2} \in E(S)$.

THEOREM 3.2. Let $S$ be a commutative categorical semigroup containing a cancellable element. Then $S$ contains an identity and $S$ is regular.

Proof. Let $x \in S$ be cancellable and $b \in S$. Since $x^{2} S$ is categorical and $x b x \in x^{2} S$, we have $x b \in x^{2} S$. Thus $x b=x^{2} y$ for some $y \in S$, which implies that $b=x y$. Therefore $S=x S$. Now $x=x t$ for some $t \in S$ and we assert that $t$ is the identity for $S$. Let $s=x z(z \in S)$ be an arbitrary element of $S$. Then

$$
s t=(x z) t=(x t) z=x z=s .
$$

From 1.2, we have that $S$ is regular.

Noting that a cancellative semigroup can contain at most one idempotent, we have the following.

COROLLARY 3.3. A commutative cancellative categorical semigroup is a group.

LemMa 3.4. Let $S$ be a commutative categorical semigroup. Then for any $a, b \in S$, there exists $x \in S$ such that $a b=a^{2} x$.

Proof. We have $a^{2} b \in a^{2} S$ and $a^{2} S$ is categorical. Hence $a b \in a^{2} S$.

Proposition 3.5. Let $S$ be a commutative categorical semigroup. Then $S^{2}$ is categorical.

Proof. Let $A$ be an ideal of $S^{2}$. It suffices to prove that $A$ is an ideal of $S$. Let $a \in A$ and $s \in S$. From 3.4, there exists $x \in S$ such that $a s=a(a x) \in$ $A S^{2} \subseteq A$.

THEOREM 3.6. Let $S$ be a commutative semigroup. Then $S$ is categorical if and only if $S$ is an extension of a regular categorical semigroup by a null semigroup.

Proof. Assume $S$ is categorical. From 3.5 and the fact that the Rees factor semigroup $S / S^{2}$ is a null semigroup, we need only prove that $S^{2}$ is regular. We show that the $\mathscr{H}$-classes of $S^{2}$ are groups and hence $S^{2}$ is a union of groups. 
Let $a, b \in S$. Then $a^{2} b \in a b S$, which implies that $a b \in a b S$. We assert that $H_{a b}$, the $\mathscr{H}$-class containing $a b$, is a group. Now there exists $x \in S$ such that $a b=a b x$. From $3.4, a b x=(a b)^{2} y$ from some $y \in S$. Thus $a b=(a b)^{2} y$ which implies that $(a b)^{2} \in H_{a b}$. Hence $H_{a b}$ is a group by Theorem 2.16 of [1].

For the converse, let $I$ be a regular categorical subsemigroup (ideal) of $S$ such that $S / I$ is null. Let $A$ be an ideal of $S$ and $a b c \in A$ with $a, b, c \in S$. Since $S / I$ is null we have $a b, b c \in I$. $I$ is regular so there exist $x, y \in I$ such that $(a b) x(a b)=a b$ and $(b c) y(b c)=b c$. Now $A I$ is an ideal of $I$ and is therefore categorical in $I$. Since $a b c \in A$, we have that $a^{2} b^{2} c^{2} \in A I$ with $a^{2}, b^{2}, c^{2} \in I$. Therefore $a^{2} b^{2} \in A I$ or $b^{2} c^{2} \in A I$. If $a^{2} b^{2} \in A I$, then $a b=a^{2} b^{2} x \in A I \subseteq A$. Similarly $b^{2} c^{2} \in A I$ implies that $b c \in A$. Therefore $A$ is a categorical ideal of $S$.

\section{REFERENCES}

1. A. H. Clifford and G. B. Preston, The algebraic theory of semigroups. Vols. 1, 2, Math. Surveys, no. 7, Amer. Math. Soc., Providence, R.I., 1961, 1967. MR 24 \#A2627; MR 36 \#1558.

2. W. D. Munn, Brandt congruences on inverse semigroups, Proc. London Math. Soc. (3) 14 (1964), 154-164. MR 30 \#3167.

3. M. Petrich, The maximal semilattice decomposition of a semigroup, Math. Z. 85 (1964), 68-82. MR 29 \#4824.

4. - On a class of completely semisimple inverse semigroups, Proc. Amer. Math. Soc. 24 (1970), 671-676. MR 41 \#372.

5. M. Satyanarayana, Principal right ideal semigroups, J. London Math. Soc. (2) 3 (1971), 549-553.

Department of Mathematics, Bowling Green State University, Bowling GREen, OHIO 43403

Current address (F. R. McMorris): Biomathematics Program, Box 5451, North Carolina State University, Raleigh, North Carolina 27607 